\title{
¿QUÉ MENSAJES DA UNA SERIE DE EJERCICIOS DE MATEMÁTICA ACERCA DE LOS ROLES DE GÉNERO?
}

\author{
QUE MENSAGENS DA UMA SÉRIE DE EXERCÍCIOS DE MATEMÁTICA \\ DÁ SOBRE PAPÉIS DE GÊNERO?
}

\section{WHICH MESSAGES DOES A SERIES OF MATHEMATICS EXERCISES GIVE ABOUT GENDER ROLES?}

\author{
Verónica Molfino \\ Consejo de Formación en Educación de Uruguay, Uruguay \\ ORCID: http://orcid.org/0000-0002-6672-762X \\ Currículo académico: https://bit.ly/34LomoY \\ Correo electrónico: veromolfino@gmail.com \\ Cristina Ochoviet \\ Consejo de Formación en Educación de Uruguay, Uruguay \\ ORCID: http://orcid.org/0000-0001-9069-3469 \\ Currículo académico: https://bit.ly/3vV921S \\ Correo electrónico: cristinaochoviet@gmail.com
}

DOI: $10.22481 /$ rbba.v10i01.8192

\section{RESUMEN}

En este trabajo se identifican los mensajes relativos a los roles de género que emergen de una serie de ejercicios de matemática elaborado para la formación de profesores de matemática que trata, específicamente, las estrategias de conteo. Desde la perspectiva de la enseñanza de la matemática para la justicia social se analizan los enunciados de los problemas poniendo el foco en los roles de género que de ellos se desprenden. Las conclusiones señalan que los enunciados de los problemas matemáticos del material 
analizado refuerzan los roles de género tradicionalmente establecidos, y dan un mensaje invisible que instituye y refuerza esos roles de género. A la vez, se alerta a los formadores a prestar atención al momento de seleccionar las actividades matemáticas que se ofrecen a los futuros profesores de matemática.

Palabras clave: Formación de profesores; Rol social; Movimiento feminista; Matemáticas.

\section{RESUMO}

Este trabalho identifica as mensagens relacionadas aos papéis de gênero que emergem de uma série de exercícios de matemática preparada para a formação de professores de matemática que lida especificamente com estratégias de contagem. Na perspectiva do ensino de matemática para a justiça social, são analisadas as declarações dos problemas, enfocando os papéis de gênero que emergem deles. As conclusões mostram que as declarações de problemas matemáticos do material analisado reforçam os papéis de gênero tradicionalmente estabelecidos e transmite uma mensagem invisível que institui e reforça esses papéis de gênero e, ao mesmo tempo, os treinadores são alertados a prestar atenção ao selecionar as atividades de matemática oferecidas aos futuros professores de matemática.

Palavras-chave: Formação de Professores; Papel social. Feminismo; Matemática.

\section{ABSTRACT}

This work identifies the messages related to gender roles that emerge from a series of mathematics exercises about counting strategies that was prepared for mathematics teacher training. From the perspective of teaching mathematics for social justice, the wording of the problems is analyzed, focusing on the gender roles that emerge from them. The conclusions indicate that the wording of the mathematical problems reinforce traditionally established gender roles, and give an invisible message that institutes and reinforces those gender roles. At the same time, trainers are alerted to pay attention when selecting the math activities offered to future math teachers. 
Keywords: Teacher training; Social role; Feminism. Mathematics.

\section{INTRODUCCIÓN}

Muchas veces la matemática es considerada una disciplina neutra que no transmite ningún tipo de sesgos acerca de la sociedad en que vivimos. Es sí usada por distintas disciplinas para analizar fenómenos y facilitar la obtención de conclusiones, pero no se considera que en sí misma transmita mensajes u opiniones acerca de una determinada temática.

Quizás la afirmación anterior pueda ser relativizada si analizamos la contextualización de las situaciones que se le ofrecen a los estudiantes para aprender matemática. En general, los docentes buscan aplicaciones interesantes o motivadoras que no estén presentadas en contexto intramatemático. Así, ingresan en el aula variados enunciados que son portadores de información que puede contener sesgos u opiniones acerca de distintos asuntos.

El objetivo de este trabajo consiste en identificar los mensajes relativos a los roles de género que emergen de una serie de ejercicios de matemática elaborada para la formación de profesores de matemática que trata, específicamente, las estrategias de conteo. La serie de ejercicios que abordamos fue elaborada por un equipo docente, en el año 2008, con el objetivo de contar con un material que estuviera disponible para los estudiantes en un plan de estudios que se comenzaba a desarrollar en ese mismo año. El material (ver Anexo) se encuentra disponible en Internet y es accesible a todos los estudiantes y docentes que son usuarios de la página del Departamento de Matemática del Consejo de Formación en Educación en Uruguayi.

\section{ANTECEDENTES TEMÁTICOS}

Los antecedentes temáticos refieren a trabajos relativos al análisis de situaciones de injusticia social que emergen de diferentes aspectos del discurso matemático escolar, como pueden ser textos, apuntes de profesores o fichas de trabajo.

Esen (2015) reporta un análisis de libros de texto de primaria diseñados acordes a una reforma curricular en Turquía. Los cambios curriculares buscan generar mayor igualdad y respeto por los derechos humanos, promoviendo la igualdad de género, raza, religión u otros atributos que generan discriminación en la sociedad turca. El equipo de trabajo de Esen centró su análisis en las imágenes de los textos de lengua turca, estudios de la vida y estudios sociales diseñados especialmente para ese nuevo currículum, focalizándose en el rol del hombre y la 


\section{¿QUÉ MENSAJES DA UNA SERIE DE EJERCICIOS DE MATEMÁTICA ACERCA DE LOS ROLES DE GÉNERO?}

mujer que esas imágenes traducían mediante ciertas categorías consideradas. La autora concluye que estos nuevos textos perpetúan la desigualdad de género y que fallan en el intento de concientizar a las nuevas generaciones sobre las discriminaciones, en particular la basada en género.

En Siti Norzaimalina, Mohamad Subakir y Bahiyah Dato’ (2011) se reporta un análisis de libros de texto de matemática en inglés para primaria en Quatar, que busca estudiar la existencia y ocurrencia de sexismo y estereotipación de género que se deducen del lenguaje empleado en los textos. El método articula herramientas cuantitativas con cualitativas, empleando el Análisis Crítico del Discurso como herramienta metodológica para los aspectos cualitativos. El estudio sobre 24 textos, algunos editados en Quatar y otros extranjeros, arrojó que, si bien aún persiste sexismo lingüístico y estereotipación de roles de género, aparecen de forma mínima, lo que los autores visualizan como una señal positiva para un país como Quatar, con fuerte tradición de discriminación de género.

Por su parte, Felton-Koestler (2017), en su amplia discusión sobre antecedentes relativos a estudios de género y otros atributos que tradicionalmente han dado lugar a discriminaciones en la enseñanza de la matemática, menciona un ejemplo de una tarea clásica propuesta a estudiantes de primaria en la que subyace una concepción binaria de género, evidenciando lo que Esmonde (2011, citado en FELTON-KOESLER, 2017) ha denominado "genderism" (p. 55).

En el contexto uruguayo existen algunos trabajos desarrollados en el área de la lingüística desde una perspectiva de género que reportan análisis de manuales escolares (GRAÑA, 2006 y 2008; ROSTAGNOL, 1993 y 1996, referidos en FURTADO, 2018). Graña (2008, referido en FURTADO, 2018) analiza manuales del período 1900-2007, evidenciando que los estereotipos de género a lo largo del tiempo se mantienen, pese a los cambios de la sociedad durante el siglo XX. Rostagnol (1993, referido en FURTADO, 2018) analiza manuales escolares vigentes en Uruguay a inicios de los años noventa, y evidencia un predominio de las figuras masculinas por sobre las femeninas, además de la reproducción de roles tradicionalmente asignados a cada género. Los hombres se asocian a roles de trabajo, estudio y juego, fuera del hogar, mientras las mujeres suelen ocuparse de tareas domésticas. "Rostagnol concluye que las representaciones propuestas por los textos escolares refuerzan los estereotipos tradicionales y que, en términos generales, el mensaje en relación al género es sexista y por consiguiente retardatario de cualquier posible cambio" (FURTADO, 2018, p. 14). 


\section{¿QUÉ MENSAJES DA UNA SERIE DE EJERCICIOS DE MATEMÁTICA ACERCA DE LOS ROLES DE GÉNERO?}

Furtado (2018) reporta un estudio sobre usos del lenguaje y representaciones sociales de género en un libro de cuarto año de primaria editado en 2008 y empleado en todas las escuelas públicas en el momento del estudio. Concluye que la perspectiva de género del texto acompaña las políticas lingüísticas del ámbito educativo y de género de la época, pero "aún se podría hacer mucho más" (FURTADO, 2018, p. 29). Si bien presenta una intencionalidad expresa de trabajar en torno a los roles estereotipados de género y presenta modelos alternativos a los tradicionales, la autora presenta variados ejemplos en los que las mujeres aún quedan excluidas de ámbitos laborales, especialmente de ámbitos científicos.

En el contexto de la Matemática Educativa, diversos estudios realizados en Uruguay se enfocan en el diseño de secuencias de enseñanza que promueven la enseñanza de la matemática para la justicia social (EMpJS). Algunos de ellos ponen la mirada, particularmente, en el análisis de temáticas de género. Por ejemplo, Dolgay y Ochoviet (2016) proponen la narración oral de cuentos como recurso didáctico para abordar la brecha salarial de género en la enseñanza media básica. A su vez, Galli, Montegui, Molfino y Núñez (2017), a través de estudios socioestadísticos, proponen una secuencia didáctica para el estudio del tema porcentajes en la enseñanza media básica y a partir de ello abren una discusión acerca de los roles tradicionales de género en la crianza de los hijos y cómo incide negativamente en la oportunidad de conseguir un trabajo el ser una madre con hijos pequeños. Estos estudios abordan dos de las perspectivas de EMpJS señaladas por Felton-Koestler (2017): conectar la matemática con la identidad social y cultural de los estudiantes y usar la matemática para el desarrollo de la conciencia crítica y el trabajo para cambiar las injusticias en nuestra sociedad.

En este trabajo realizamos un abordaje no estudiado anteriormente, que consiste en analizar qué mensaje dan sobre los roles de género, los enunciados de un conjunto de ejercicios de matemática sobre estrategias de conteo.

\section{PERSPECTIVA TEÓRICA}

Los inicios de la pedagogía crítica son asociados a los trabajos que Paulo Freire desarrolló a partir de 1963 sobre alfabetización y pedagogía de la liberación (STINSON; BIDWELL; POWELL, 2012). Varios autores vinculan estos trabajos con los fundamentos de la EMpJS (GONZÁLEZ, 2009, GUTSTEIN, 2006, LÓPEZ; GUERRA, 2017, Wright, 2014).

Felton-Koestler (2017) afirma que los trabajos con enfoque en la EMpJS comienzan a desarrollarse incipientemente en la última década del siglo XX y cobran impulso en la primera 


\section{¿QUÉ MENSAJES DA UNA SERIE DE EJERCICIOS DE MATEMÁTICA ACERCA DE LOS ROLES DE GÉNERO?}

quincena del siglo XXI. Según este autor las discusiones en torno a la EMpJS y la educación matemática crítica abarcan muy diversas perspectivas que, no obstante, tienen en común la consideración de dos conjuntos de objetivos pedagógicos dialécticamente relacionados: uno relativo a la justicia social y el otro a la matemática (GUTSTEIN, 2006).

Como muchos autores señalan, introducir la perspectiva de la EMpJS es un gran desafío, especialmente en programas de formación de profesores (FELTON-KOESTLER, 2017, AGUIRRE, 2009, ENSIGN, 2005).

Felton-Koestler (2017) introduce el marco Qué, Quién y Cómo (WWH por su sigla en inglés) para comprender la visión de los profesores y su propia instrucción como docentes al enseñar bajo la perspectiva de la EMpJS. Este marco permite informar acerca de la enseñanza de la matemática, considerando las creencias de los profesores y la naturaleza de la matemática escolar.

La EMpJS tiene un claro foco en la sociedad o el medio en el que vivimos y por ello, el WWH se concentra en las relaciones entre la matemática que se enseña en las instituciones educativas y el mundo en el que las instituciones están insertas, entendiendo por mundo, o también mundo real, el contexto sociopolítico de la institución de referencia. Felton-Koestler (2017, p. 50-51) se centra en tres preguntas clave: ¿Qué mensajes da la enseñanza de la matemática?

En particular, se pregunta sobre qué mensajes damos sobre la consideración de la matemática como coconstruida junto a otros constructos sociales como raza, género, clase, cultura u otros marcadores de diferencia social; y también qué mensajes da sobre el rol del análisis social en la matemática escolar (el rol de la matemática para comprender, analizar y criticar nuestro mundo).

- ¿De quiénes son las perspectivas que deberían estar representadas en la matemática escolar y de qué manera se relacionan con los estudiantes?

- ¿Cómo deberían estar relacionados los conceptos matemáticos con el mundo real en la matemática escolar?

Según el autor estas preguntas son lo suficientemente amplias como para permitir poner la atención en una variedad de situaciones educativas.

En este estudio nos focalizamos en la primera de las preguntas, las otras dos preguntas las utilizaremos para elaborar recomendaciones a partir del presente estudio. En particular en 


\section{¿QUÉ MENSAJES DA UNA SERIE DE EJERCICIOS DE MATEMÁTICA ACERCA DE LOS ROLES DE GÉNERO?}

la relación entre la matemática escolar y el análisis social. Al respecto, Koestler (2012, p. 59) señala que:

Los temas que un profesor incluye (o no), las actividades que pide hacer a los estudiantes y las formas de participación que les solicita, todo eso envía mensajes a los estudiantes sobre lo que es importante, válido y valorado en matemática y en la escuela... Con respecto a las maneras en que los profesores presentan el rol de las matemáticas en el mundo, ellos pueden enfatizar contextos de uso frecuente en enseñanza primaria, como manzanas, perros o helados, o pueden incluir problemas que emplean la matemática para comprender y analizar cuestiones urgentes como temas medioambientales o la participación democrática (referido en FELTON-KOESTLER, 2017, p. 59).

En concreto, en este estudio nos interesa conocer ¿Qué mensajes dan acerca de los roles de género los enunciados de una serie de ejercicios sobre estrategias de conteo en una propuesta de enseñanza para un curso del profesorado de Matemáticas?

Con el objetivo de construir una herramienta analítica que nos permita discriminar la existencia de mensajes con sesgo de género, consideraremos las relaciones entre la matemática y la comprensión del mundo que propone Felton-Koestler (2017), pues nos permitirán identificar los contextos en que son propuestos los ejercicios que nos proponemos analizar.

Este autor ha identificado cuatro tipos de relaciones entre la matemática y la comprensión del mundo:

1. Disciplina distinta: La matemática es una disciplina particular, prácticamente autocontenida, que no se relaciona con la vida cotidiana o el entorno y no se ocupa de temáticas sociales o políticas.

2. Mundo real: La matemática debe estar vinculada a temáticas del mundo en el que vivimos que son apolíticas o neutrales. No toma posiciones acerca de las problemáticas sociales.

3. Sociopolítica: La matemática debe estar relacionada a temas claramente políticos o a asuntos controversiales.

4. Injusticia: La matemática debe estar conectada a temas que pretenden generar conciencia para comprender y trabajar para la superación de las injusticias que la disciplina permite identificar.

El autor aclara que estas visiones no constituyen categorías estancas, sino que los docentes o los estudiantes de profesorado podrán ubicarse en una de estas visiones o transitar entre ellas. 


\section{¿QUÉ MENSAJES DA UNA SERIE DE EJERCICIOS DE MATEMÁTICA ACERCA DE LOS ROLES DE GÉNERO?}

\section{MÉTODO}

Este estúdio es de carácter teórico y cualitativo, y pretende indagar qué mensajes se desprenden de los enunciados de los problemas matemáticos incluidos en una serie de ejercicios para la formación de profesores de matemática en Uruguay. En concreto, se trata de un material preparado para la asignatura Fundamentos de la Matemática, correspondiente al primer año de la formación de profesores. En Uruguay, la carrera de profesor de matemática consta de cuatro años, y esta asignatura es una de las matemáticas que se estudian en el primer año de la carrera. Refiere, en general, a temas del álgebra.

El material analizado está dividido en dos grandes secciones. La primera contiene el desarrollo teórico de los temas y ejemplos. Aborda los siguientes contenidos en seis secciones: principio de la adición y la multiplicación, combinaciones, propiedades de los números combinatorios, principio del palomar, principio de inclusión-exclusión, números de Stirling de segunda especie. La segunda parte presenta la sección 7: Ejercicios, y contiene 34 problemas de aplicación de los conceptos estudiados. En este trabajo se consideran los problemas propuestos en esta sección y, dentro de esta, se analizarán los problemas que están planteados en contexto_reformativos_os y, además, aquellos en los que en los enunciados se alude específicamente a hombres, mujeres, se utiliza el nombre de la persona, o bien aquellas situaciones en las que aparece una sola persona y entonces es necesaria la discriminación de género. No se consideran aquellos enunciados que utilizan plurales como políticos, médicos, etcétera, dado que desde el punto de vista formal del uso del español no es necesaria la discriminación de género y, además, no era de uso el lenguaje inclusivo en el año 2008 en Uruguay, momento en que se elaboró el material que se analiza. De esta manera, cuidamos que no se establezcan sesgos en el análisis, que si bien podían estar manifiestos por creencias de los profesores que elaboraron el material, no son legítimamente evidenciables a través de ese uso idiomático.

Es decir que nuestro estudio no se enfoca en los usos del lenguaje, ya sean inclusivos o no inclusivos, sino en las representaciones sociales de género (FURTADO, 2018). Coincidimos con la autora en la importancia de "identificar a los roles tradicionales como un problema central, transmitidos por la sociedad patriarcal a niñas y mujeres a través de la educación, la familia, los medios de comunicación y las prácticas culturales y lingüísticas" (FURTADO, 2018, p. 22). 
ACERCA DE LOS ROLES DE GÉNERO?

\section{RESULTADOS}

A continuación, presentamos cinco categorías que resumen los distintos contextos en que son presentados cada uno de los problemas de la sección Ejercicios e identificamos cuáles de ellos son materia de análisis de este trabajo.

\section{Tabla 1 - Distribución de los ejercicios de la serie en categorías contextuales}

\begin{tabular}{|c|c|c|c|}
\hline Categoría & Ordinal del problema & Ejemplo & Seleccionados \\
\hline $\begin{array}{l}\text { Contexto } \\
\text { intramatemático }\end{array}$ & $\begin{array}{c}8,12,13,15,16,20,21 \\
22,24\end{array}$ & $\begin{array}{l}\text { En una circunferencia se } \\
\text { ubican seis puntos. } \\
\text { ¿Cuántos segmentos } \\
\text { determinan? }\end{array}$ & \\
\hline $\begin{array}{l}\text { Contexto } \\
\text { extramatemático que no } \\
\text { alude a personas }\end{array}$ & $\begin{array}{c}1,4,5,9,10,11,14,17 \\
25,28,29,30,32\end{array}$ & $\begin{array}{l}\text { En un torneo de fútbol, } \\
\text { participan } 10 \text { equipos. } \\
\text { Cada uno juega con los } \\
\text { demás equipos } \\
\text { dos veces. ¿Cuántos } \\
\text { partidos se juegan? }\end{array}$ & \\
\hline $\begin{array}{l}\text { Contexto } \\
\text { extramatemático que } \\
\text { alude a personas con } \\
\text { plurales genéricos } \\
\text { masculinos }\end{array}$ & $2,19,23,27$ & $\begin{array}{l}\text { Cinco políticos se } \\
\text { encuentran en una fiesta. } \\
\text { ¿Cuántos saludos de } \\
\text { mano se } \\
\text { intercambian si cada } \\
\text { uno estrecha la mano de } \\
\text { los demás sólo una vez? }\end{array}$ & \\
\hline $\begin{array}{l}\text { Contexto } \\
\text { extramatemático que } \\
\text { alude a personas con } \\
\text { plurales genéricos } \\
\text { femeninos } \\
\text { discriminación } \\
\text { género }\end{array}$ & 26,31 & $\begin{array}{l}\text { Un auditorio tiene } \\
\text { capacidad para } 800 \\
\text { personas. ¿Cuántos } \\
\text { asientos deben ocuparse } \\
\text { para garantizar que al } \\
\text { menos dos personas } \\
\text { sentadas en el auditorio } \\
\text { tienen las mismas } \\
\text { iniciales del nombre y } \\
\text { del primer apellido? }\end{array}$ & \\
\hline $\begin{array}{l}\text { Contexto } \\
\text { extramatemático que } \\
\text { alude a personas } \\
\text { específicas, r con } \\
\text { discriminación } \\
\text { género }\end{array}$ & $3,6,7,18,33,34$ & $\begin{array}{l}\text { Luis y Mario juegan un } \\
\text { torneo de tenis. El } \\
\text { tenista que gane dos } \\
\text { partidas consecutivas } \\
\text { o triunfe en tres, gana el } \\
\text { torneo. ¿Cuáles son los } \\
\text { resultados posibles de la } \\
\text { competencia? }\end{array}$ & $\mathrm{X}$ \\
\hline
\end{tabular}

Fuente: Elaboración propia.

En suma, identificamos: nueve ejercicios con contexto intramatemático (por ejemplo, "En una circunferencia"), trece ejercicios con contexto extramatemático que no alude a 


\section{¿QUÉ MENSAJES DA UNA SERIE DE EJERCICIOS DE MATEMÁTICA ACERCA DE LOS ROLES DE GÉNERO?}

personas (por ejemplo, "participan 10 equipos"), seis ejercicios con contexto extramatemático que alude a personas con plurales genéricos masculinos o femeninos (por ejemplo, "Cinco políticos" o "las personas", respectivamente) y seis ejercicios con contexto extramatemático que alude a personas específicas, con discriminación de género (por ejemplo, "Luis y Mario juegan al tenis"). Son estos últimos seis ejercicios los que nos pueden informan acerca de: ¿qué mensajes dan sobre la discriminación de género los enunciados de una serie de ejercicios sobre estrategias de conteo?

Dentro de esos ejercicios, tenemos aquellos que refieren a hombres en relación a su interés por los deportes (ejercicios 3 y 34) o sus trabajos, relativos a computadoras u oficios tradicionalmente asignados a los hombres como pintor o cortador de césped (ejercicios 7, 18 y 33). Los ejercicios que refieren a mujeres son dos: en el ejercicio 6 las mujeres participan en el contexto como personas que "van de compras", es decir, se les asigna un rol de consumidoras en lugar de productoras, no son quienes trabajan sino quienes gastan el dinero. Mientras tanto, en el mismo ejercicio, se asocia a los hombres a posibles hechos delictivos: "Un sábado, cuando iban de compras, Juana y Teresa vieron a dos hombres alejarse en automóvil de la fachada de una joyería, justo antes de que sonara la alarma contra robos" (p. 24-25). En el ejercicio 34, la señora Báez es la encargada de comprar los regalos de Navidad, nuevamente se le asigna un rol pasivo en cuanto a su desempeño profesional o sus intereses personales.

Respecto a algún otro elemento en el documento que nos permita decir algo acerca de los mensajes que da la enseñanza de la matemática mediante el material que analizamos, podemos apuntar dos cuestiones que están en secciones anteriores: una es relativa a un hombre que integra un equipo: "Juan integra un equipo de 5 personas que deciden formar una comisión de tres para organizar una reunión a fin de año" (Técnicas de conteo, p. 15). Si bien este enunciado trata sobre un hombre, no detectamos en él una discriminación específica sobre el género, ya que no se indica si el equipo es de deporte, de trabajo o simplemente un equipo de amigos, tampoco se explica si es mixto o sólo formado por hombres, por lo que no nos aporta información respecto al rol que se le asigna a la mujer o al hombre en la sociedad. La otra observación que hacemos relativa a secciones anteriores a la 7, es en la página 14: "Laura realiza un posgrado en Educación Matemática”. Ese es el único contexto en el que se muestra a una mujer en un rol activo, vinculado con su desarrollo profesional. 


\section{CONCLUSIONES}

Comenzamos estas conclusiones con el resultado de un ejercicio teórico que podría tener por enunciado "construye un relato de lo que el material de estudio informa sobre roles asignados a diferentes géneros en la sociedad". Guiándonos por los ejercicios que figuran en la sección 7, elaboramos dicho relato:

A las mujeres les gusta ir de compras mientras que a los varones les gustan los
deportes. Los varones pueden realizar las tareas domésticas asignadas
tradicionalmente a los hombres, como pintar una pared o cortar el césped. Los
varones trabajan con computadoras. Hay varones que son sospechosos de
hurtos. Las mujeres se encargan de comprar los regalos de Navidad para sus
hijos.

El análisis realizado sobre el material de estudio nos permite, entonces, poner de relieve que, a pesar de que la matemática es tradicionalmente concebida como "una asignatura libre de valores, neutral y basada solamente en el pensamiento lógico y razonamiento deductivo" (D’AMBROSIO, 2008, referido en WRIGHT, 2014, p. 2), su enseñanza emite ciertos mensajes. En este estudio se identificaron, específicamente, mensajes referidos a los diferentes roles que asumen mujeres y varones en la sociedad, y los que les son atribuidos. Los enunciados de los problemas de este material de estudio sobre técnicas de conteo revelan las expectativas sociales acerca de los roles de género y podrían contribuir a sedimentar la reproducción de ciertas formas de actuar de los cuerpos (BUTLER, 1998).

Así, este trabajo oficia como una alerta a los equipos docentes al momento de seleccionar los enunciados de los problemas que se propondrán a los estudiantes, futuros profesores de matemática en este caso, pues el conjunto de problemas y las temáticas a las que se hace referencia en ellos comunican a los estudiantes determinados roles de género que al no ser materia de análisis en forma explícita ofician como relatos invisibles que sin duda abonan a la construcción de las identidades de género y a la institucionalización de los roles de género predominantes en nuestra sociedad.

\section{REFERENCIAS}

AGUIRRE, J. M. Privileging mathematics and equity in teacher education: Framework, counterresistance strategies, and reflections from a Latina mathematics educator. En:

B. GREER; S. MUKHOPADHYAY; A. B. POWELL; S. NELSON-BARBER (Eds.), Culturally responsive mathematics education. New York: Routledge, 2009, p. 295-319. 
BUTLER, J. Actos performativos y constitución del género: un ensayo sobre fenomenología y teoría feminista. Debate feminista, México D.F., v. 18, p. 296-314, oct. 1998.

DOLGAY, M.; OCHOVIET, C. Una historia de contadores. En: G. BUENDÍA; V. MOLFINO; C. OCHOVIET (Comps.), Estrechando lazos entre investigación y formación en Matemática Educativa. Volumen III. Montevideo: CFE, 2016, p. 43-50.

ENSIGN, J. Helping teachers use students' home cultures in mathematics lessons: Developmental stages of becoming effective teachers of diverse students. En: A. J. RODRIGUEZ; R. S. KITCHEN (Eds.), Preparing mathematics and science teachers for diverse classrooms: Promising strategies for transformative pedagogy. Mahwah, NJ: Lawrence Erlbaum Associates, 2005, p. 225-242.

ESEN, Y. Sexism in School Textbooks Prepared under Education Reform in Turkey. Journal for Critical Education Policy Studies, Athens, v. 5, n. 2, p. 466-493, nov. 2007.

FELTON-KOESTLER, M. Mathematics education as sociopolitical: prospective teachers' views of the What, Who, and How. Journal of Mathematics Teacher Education, Netherlands, v. 20, n. 1, p. 49-74, feb. 2017.

FURTADO, V. De niñas y niños: las políticas lingüísticas de género en la educación primaria uruguaya. Lingüística, v. 34, n. 2, p. 9-31, dic. 2018.

GALLI, M.; MOLFINO, V.; MONTEGUI, E.; NÚÑEZ, I. Desnaturalizando lo socialmente establecido: una discusión de género. En: G. BUENDÍA; V. MOLFINO; C. OCHOVIET (Comps.), Estrechando lazos entre investigación y formación en Matemática Educativa. Volumen IV. Montevideo: CFE, 2017, p. 85-96.

GRIMALDI, R. Matemáticas discreta y combinatoria: Una introducción con aplicaciones. México: Pearson Educación, 1997.

GONZALEZ, L. Teaching mathematics for social justice: Reflections on a community of practice for urban high school mathematics teachers. Journal for Urban Mathematics Education, v. 2, n. 1, p. 22-51, jul. 2009. Disponible en: <http://edosprey.gsu.edu/ojs/index.php/JUME/article/view/32/13. Acceso: 14. mar. 2021.>

GUTSTEIN, E. Reading and writing the world with mathematics: Toward a pedagogy for social justice. New York: Routledge, 2006.

LÓPEZ, R.; GUERRA, P. Enseñanza de la Matemática para la Justicia Social. Experiencia IFD de Pando - Universidad de Kennesaw, EEUU. En:

de Educación Matemática, p. 245-252, 2017. Disponible en: http://semur.edu.uy/curem/actas/pdf/56.pdf. Acceso: 14. mar. 2021.

OSÍN, L. Introducción al análisis matemático. Buenos Aires: Kapelusz, 1966. 


\section{¿QUÉ MENSAJES DA UNA SERIE DE EJERCICIOS DE MATEMÁTICA ACERCA DE LOS ROLES DE GÉNERO?}

SITI NORZAIMALINA, A. M.; MOHAMAD SUBAKIR, M. Y.; BAHIYAH DATO', A. H. Linguistics Sexism in Mathematics Textbooks in Qatar Primary Schools. SoLLs.INTEC 2011 Proceedings, p. 1-12, 2011. Universiti Kebangsaan Malaysia.

STINSON, D.; BIDWELL, C.; POWELL, G. Critical pedagogy and teaching mathematics for social justice. The International Journal of Critical Pedagogy, v.4, n. 1, p. 76-94, 2012. Disponible en: <http://libjournal.uncg.edu/ojs/index.php/ijcp/article/view/302/263>. Acceso: 14. mar. 2021.

TÉCNICAS DE CONTEO. Material para los estudiantes. Montevideo: Departamento de Matemática, Consejo de Formación en Educación, 2008. Disponible en: <http://www.depdematematica.org/ipa/sitio/>. Acceso: 14. mar. 2021.

WRIGHT, P. (2014). Teacher researchers, mathematics classrooms and social justice. Presentación en BERA Conference 2014. Londres, 2014. Disponible en: <http://mathssocialjustice.weebly.com/uploads/3/0/2/7/30279643/wright_2014_bera_paper.pdf.> Acceso: 14. mar. 2021.

\section{ANEXO}

\section{Ejercicios}

(1) Los automóviles Buick se fabrican en 4 modelos, 12 colores, 3 tamaños de motor y 2 tipos de transmisión.

a) ¿Cuántos Buick distintos pueden fabricarse?

b) Si uno de los colores disponibles es el azul, ¿cuántos Buick azules diferentes se pueden fabricar?

(2) a) El consejo directivo de una empresa farmacéutica tiene 10 miembros. Se ha programado una próxima reunión de accionistas para aprobar una nueva lista de ejecutivos (elegidos entre los diez miembros del consejo). ¿Cuántas listas diferentes, formadas por un presidente, un vicepresidente, un secretario y un tesorero, puede presentar el consejo a los accionistas para su aprobación?

b) Tres miembros del consejo de directores (de la parte anterior) son médicos.

¿Cuántas listas de la parte anterior tienen

i) un médico nominado para la presidencia?

ii) exactamente un médico en la lista?

iii) al menos un médico en la lista?

(3) Luis y Mario juegan un torneo de tenis. El tenista que gane dos partidas consecutivas o triunfe en tres, gana el torneo. ¿Cuáles son los resultados posibles de la competencia? 


\section{¿QUÉ MENSAJES DA UNA SERIE DE EJERCICIOS DE MATEMÁTICA ACERCA DE LOS ROLES DE GÉNERO?}

(4) En un torneo de fútbol, participan 10 equipos. Cada uno juega con los demás equipos dos veces. ¿Cuántos partidos se juegan?

(5) De la ciudad A a la ciudad B hay cinco caminos, y de la ciudad B a la C hay tres caminos.

a) ¿De cuántas maneras se puede ir de $\mathrm{A}$ a $\mathrm{C}$ pasando por $\mathrm{B}$ ?

b) ¿De cuántas maneras se puede hacer el trayecto: A-B-C-B-A?

c) ¿De cuántas maneras se puede hacer el trayecto: A-B-C-B-A si la ruta de regreso BA no puede ser la misma que la elegida para ir de A a B?

(6) Un sábado, cuando iban de compras, Juana y Teresa vieron a dos hombres alejarse en automóvil de la fachada de una joyería, justo antes de que sonara la alarma contra robos. Aunque todo ocurrió muy rápido, cuando fueron interrogadas las dos jóvenes, pudieron dar a la policía la siguiente información acerca de la placa (que constaba de dos letras seguidas de cuatro dígitos) del automóvil que huyó. Teresa estaba segura de que la segunda letra de la placa era una $\mathrm{O}$ o una $\mathrm{Q}$, y que el último dígito era un 3 o un 8. Juana dijo que la primera letra de la placa era una $\mathrm{C}$ o una $\mathrm{G}$ y que el primer dígito era definitivamente un 7. ¿Cuántas placas diferentes tendrá que verificar la policía?

(7) Matías trabaja como operador de computador en una pequeña universidad. Una tarde, él ve que durante el día se han enviado 12 programas para su procesamiento por lotes. ¿De cuántas formas puede ordenar Matías el procesamiento de estos programas si:

(a) no existen restricciones?

(b) él considera que cuatro programas tienen prioridad sobre los otros ocho y desea procesarlos antes?

(c) primero separa los programas en los cuatro de máxima prioridad, cinco de menor prioridad y tres de mínima prioridad, y desea procesar los 12 programas de modo que los de máxima prioridad se procesen primero y los tres programas de mínima prioridad se procesen al final?

(8) En una circunferencia se ubican seis puntos. ¿Cuántos segmentos determinan?

(9) Usando letras sin repetir de la palabra MURCIÉLAGO, hallar:

a) de cuántas maneras puede elegirse una consonante y una vocal.

b) cuántas palabras (con o sin sentido) pueden formarse con una consonante y una vocal.

c) cuántas palabras de 5 letras pueden escribirse.

d) cuántas de las anteriores comienzan con M

e) cuántas de las del caso c contienen M 


\section{¿QUÉ MENSAJES DA UNA SERIE DE EJERCICIOS DE MATEMÁTICA ACERCA DE LOS ROLES DE GÉNERO?}

f) cuántas de las de c) comienzan con $\mathrm{M}$ y terminan con $\mathrm{O}$

g) en cuántas de c) aparecen juntas $\mathrm{M}$ y $\mathrm{O}$

h) en cuántas de c) aparecen juntas M, O y U

i) en cuántas de c) aparece el vocablo MOU

(10) ¿De cuántos modos se pueden disponer 10 libros en un estante, si 4 de ellos ocupan lugares fijos aunque intercambiables entre sí?

(11) Se dispone de 4 banderines triangulares de diferente color y de 3 juegos iguales compuestos cada uno por 9 banderas rectangulares distintas; se efectúan señales izando simultáneamente un banderín triangular seguido de $0,1,2$ o 3 banderas rectangulares.

a) ¿Cuántas señales diferentes pueden hacerse?

b) desde el punto de vista de la cantidad de señales, ¿qué es más conveniente perder: 3 banderas del mismo color, o dos juegos completos de banderas rectangulares?

(12) a) ¿Cuántos números de 5 cifras sin repetir pueden escribirse con los dígitos?

b) ¿Cuántos de ellos comienzan con 2 ?

c) ¿en cuántos de los de b) ocupa el 5 el tercer lugar?

d) ¿cuántos son menores que 90000 ?

e) ¿cuántos son menores que 89763 ?

(13) Hallar cuántos productos distintos pueden formarse con 5 factores sin repetir elegidos entre los elementos de los conjuntos:

a) $\{1,2,3,5,7,11,13,17\}$

b) $\{1,2,3,4,6,9,11\}$

(14) ¿De cuántas maneras pueden ser ordenadas 10 pruebas de examen de modo que la mejor y la peor nunca están juntas? ¿En cuántos de los casos anteriores hay 4 pruebas interpuestas entre la mejor y la peor?

(15) Hallar la suma de todos los números que se pueden obtener permutando las cifras del número 123456.

(16) Hallar la suma de todos los números mayores de 10000 que se pueden formar utilizando las cifras $0,2,4,6,8$ sin que ninguna se repita.

(17) ¿De cuántas maneras se pueden repartir 7 juguetes distintos entre 4 niños, de modo que a cada niño le corresponda un juguete por lo menos?

(18) Un pintor dispone de diez colores distintos y desea mezclar cinco de ellos en igual cantidad. 
a) ¿Cuántas mezclas distintas se pueden formar?

b) ¿Cuántas mezclas se pueden hacer si deben estar dos colores de esos diez?

c) ¿Cuántas mezclas se pueden hacer si no deben estar tres de los diez?

d) ¿Cuántas mezclas se pueden hacer si se cumplen (b) y (c) a la vez?

(19) Ocho estudiantes disponen de tres computadoras para realizar unos trabajos. El profesor les comunica que en la máquina $\mathrm{N}^{\mathrm{o}} 1$ sólo pueden trabajar tres estudiantes, en la $\mathrm{N}^{\mathrm{o}} 2$ otros tres estudiantes, y con la $\mathrm{N}^{\circ} 3$, solamente pueden trabajar dos estudiantes. ¿De cuántas maneras distintas pueden distribuirse los ocho estudiantes?

(20) Halla el número de rectas que pasan por cuatro puntos del plano no alineados 3 a 3.

(21) ¿Cuántas diagonales tiene un dodecágono? ¿Y un polígono de $n$ lados?

(22) ¿Qué polígono tiene el mismo número de diagonales que de lados?

(23) Cinco políticos se encuentran en una fiesta. ¿Cuántos saludos de mano se intercambian si cada uno estrecha la mano de los demás sólo una vez?

(24) ¿Cuántos números mayores que 1.000 .000 pueden escribirse utilizando las cifras 0,4 ,

\section{$4,5,5,5,7$ ?}

(25) La imagen corresponde al plano de una ciudad, en la cual se desea ir desde la esquina A hasta la esquina B. ¿De cuántas maneras puede hacerse ese recorrido, de modo que nunca se retroceda?

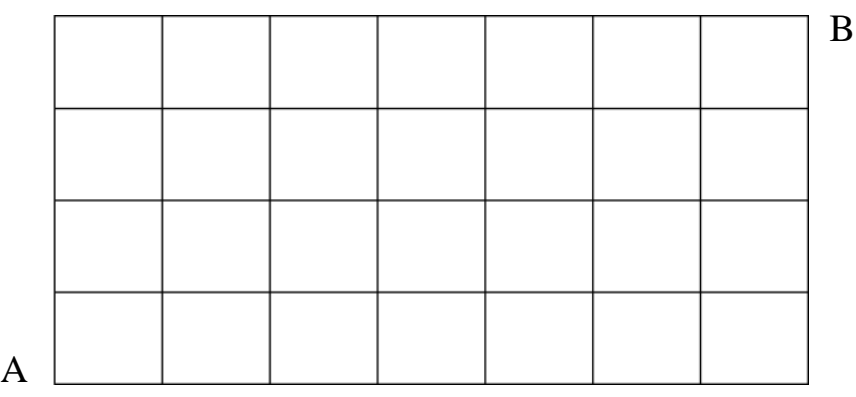

(26) ¿De cuántas maneras diferentes pueden sentarse seis personas alrededor de una mesa redonda?

(27) ¿De cuántas formas es posible distribuir 10 monedas (idénticas) entre cinco niños si (a) no hay restricciones? (b) cada niño recibe al menos una moneda? (c) el niño mayor recibe al menos dos monedas? 


\section{¿QUÉ MENSAJES DA UNA SERIE DE EJERCICIOS DE MATEMÁTICA ACERCA DE LOS ROLES DE GÉNERO?}

(28) ¿De cuántas maneras se pueden distribuir ocho bolas blancas idénticas en cuatro recipientes distintos de modo que (a) ningún recipiente quede vacío? (b) el cuarto recipiente contenga un número impar de bolas?

(29) Una moneda se lanza 60 veces, dando como resultado 45 caras y 15 cruces. ¿De cuántas maneras podría haber ocurrido esto de modo que no hubiera cruces consecutivas?

(30) ¿Cuántas veces debemos tirar un sólo dado para asegurarnos de obtener el mismo número...

a) al menos dos veces?

b) al menos tres veces?

c) al menos $n$ veces, para $n \geq 4$ ?

(31) Un auditorio tiene capacidad para 800 personas. ¿Cuántos asientos deben ocuparse para garantizar que al menos dos personas sentadas en el auditorio tienen las mismas iniciales del nombre y del primer apellido?

(32) Supongamos que tenemos siete piedras de diferente color, y cuatro recipientes numerados I, II, III y IV.

a) ¿De cuántas formas podemos distribuir las piedras de modo que ningún recipiente quede vacío?

b) En esta colección de siete piedras de colores diferentes, una de ellas es azul. ¿De cuántas formas podemos distribuir las piedras de modo que ningún recipiente quede vacío y que la piedra azul quede en el recipiente II?

c) Si eliminamos los números de los recipientes de modo que ya no podamos distinguirlos, ¿de cuántas formas podemos distribuir las siete piedras de color entre los cuatro recipientes idénticos, de modo que alguno o algunos de ellos puedan quedar vacíos?

(33) Como sus dos padres trabajan, Tomás, Eduardo y Carlos deben encargarse de las diez tareas semanales de la casa.

a) ¿De cuántas maneras pueden dividirse las tareas de tal manera que cada uno sea responsable de al menos una de ellas?

b) ¿De cuántas maneras pueden asignarse las tareas si Tomás, por ser el mayor, debe cortar el césped (una de las diez tareas semanales) y a ninguno se le permite estar ocioso?

(34) La señora Báez tiene cinco hijos (Miguel, Ricardo, David, Enrique y Donaldo) a quienes les gusta leer libros de deportes. Como se acerca la Navidad, ella visita una librería donde encuentra 12 libros diferentes de deportes. 


\section{¿QUÉ MENSAJES DA UNA SERIE DE EJERCICIOS DE MATEMÁTICA ACERCA DE LOS ROLES DE GÉNERO?}

a) ¿De cuántas formas puede seleccionar nueve de estos libros?

b) Después de hacer su compra, ¿de cuántas formas puede distribuir los libros entre sus hijos de modo que cada uno de ellos reciba al menos un libro?

c) Dos de los nueve libros que la señora Báez eligió son de baloncesto, el deporte favorito de Donaldo. ¿De cuántas formas puede ella distribuir los libros entre sus hijos para que Donaldo obtenga al menos ambos libros de baloncesto?

\section{NOTAS}

${ }^{\text {i }}$ Acerca de, ver: http://www.depdematematica.org/ipa/sitio/ 